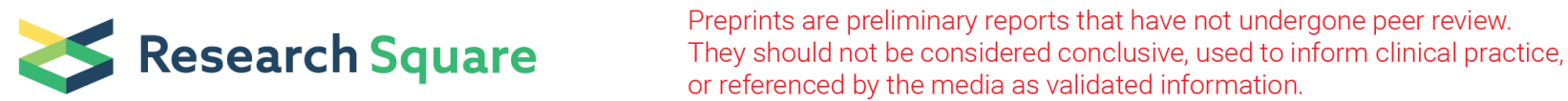

\section{Continuum of Maternal and Newborn Health in Sierra Leone: a 2019 National Survey}

Quraish Sserwanja ( $\nabla$ qura661@gmail.com )

GOAL Global

\section{Linet M. Mutisya}

Swedish Organization for Global Health

Lilian Nuwabaine

Aga Khan University

Kassim Kamara

Ministry of Health and sanitation

Ronald K Mutebi

Makerere University

Milton W. Musaba

Mbale Regional Referral and Teaching Hospital

\section{Research Article}

Keywords: Continuum of care, Sierra Leone, DHS, maternal care

Posted Date: December 22nd, 2021

DOI: https://doi.org/10.21203/rs.3.rs-1068242/v1

License: (c) (i) This work is licensed under a Creative Commons Attribution 4.0 International License.

Read Full License 


\section{Abstract}

\section{Introduction}

Globally, Sierra Leone has some of the worst maternal and child health indicators. The situation is worsened by a dearth of evidence about the level of continuum of care, an evidence-based intervention aimed at reducing maternal and perinatal morbidity and mortality. Hence this study aimed to fill this gap in evidence in the level of and factors associated with continuum of maternal and newborn care.

\section{Method}

The study employed data from the 2019 Sierra Leone Demographic Health Survey (SLDHS). Analysis was restricted to women who had a live birth in the five years preceding the survey $(n=7,326)$. Bi-variable and multivariable logistic regression were performed using SPSS software version 25.

\section{Results}

Only $17.9 \%$ (95\% Cl: $17.4-19.1)$ of the women utilized complete continuum of care (CoC) for maternal and newborn health services in Sierra Leone. About 22\% (95\% Cl: 21.3-23.1) utilized 8 or more antenatal care (ANC) contacts, 88\% (95\% Cl: 87.9-89.4) had skilled birth attendance (SBA) while 90.7\% (95\% Cl: 90.291.5) and $90.4 \%$ (95\% Cl: 89.9-91.2) of mothers and neonates utilized postnatal care (PNC) respectively. Having started ANC within first trimester (aOR 1.71, 95\% Cl: 1.46-2.00), belonging to the Southern region (aOR 1.85, 95\% Cl: 1.23-2.80), belonging to richer wealth quintile (aOR 1.76, 95\% Cl: 1.27-2.44), using internet (aOR 1.49, 95\% Cl: 1.12-1.98) and having no big problems seeking permission to access healthcare (aOR 1.34, 95\% Cl: 1.06-1.69) were significantly associated with utilization of CoC.

\section{Conclusion}

The overall completion of continuum of maternal care is low, with ANC being the lowest utilized component of continuum of care. The study findings further call for urgent attention for maternal health stakeholders to develop and implement tailored interventions prioritizing women empowerment, access to affordable internet services, timely initiation of ANC contacts, women in developed regions such as the Western and those from poor households.

\section{Introduction}

Globally, Maternal and child health $(\mathrm{MCH})$ is still a key public health priority because many countries risk missing the sustainable development goal (SDG) 3 targets of reducing maternal mortality ratio (MMR) and neonatal mortality ratio (NMR) to less than 70 deaths per 100,000 live and 12 per 1,000 live births respectively by $2030[1,2]$. Many countries across the globe continue to grapple with high maternal and perinatal mortality rates [3]. Estimates indicate that over 810 women die due to maternal related complications every day, with over $94 \%$ of these deaths occurring in low and middle income countries [4]. Sub-Saharan Africa alone accounts for approximately $67 \%$ of the maternal deaths with over 462 deaths 
per 100,000 live births [5]. The estimated neonatal mortality rate indicates that $27 \cdot 8$ million children die globally in their first 28 days of life and still the highest rate remains in sub Saharan Africa [6].

Even within sub-Saharan Africa, major disparities exist, Sierra Leone not only remains one of the poorest countries, it also reports some of the highest maternal and child indicators [7-9]. For instance, the maternal mortality ratio (MMR) stands at 717 deaths per 100,000 live births, while the neonatal mortality ratio (NMR) is 31 deaths per 1,000 live births respectively $[10,11]$.

The World Health Organization has recommended a number of interventions and strategies to strengthen the continuum of care and these have been adopted by many countries including Sierra Leone [5, 14]. These include integrated and uninterrupted antenatal care (ANC), skill birth attendance (SBA), and postnatal care (PNC) packages as critical strategies in reducing maternal and neonatal mortality and morbidity [1]. Timely ANC contacts are meant to effect birth preparedness, enable identification and treatment of illness during pregnancy [15]. Having skilled birth attendance from qualified and experienced professionals assures safe delivery with the necessary drugs and supplies for effective prevention, management of emergencies and also for referral in case of any obstetric complications $[16,17]$. Overall, completion of the continuum of care follows a pathway from pregnancy to delivery to postpartum, where each step adds a value to ensure better health outcomes for mothers and newborns, and also contributes in maternal and neonatal mortality reduction $[11,13]$.

Optimal access to a complete of continuum of care has been proposed as a possible evidence-based intervention aimed at reducing preventable maternal and perinatal deaths [1]. The concept of maternal and newborn continuum of care is grounded in the assumption that well-being and health of women and newborns should be closely linked and managed in a unified way [12]. In addition to saving lives of mothers, completion of the continuum can save up to 160,000 newborn lives by maximizing ANC coverage, 390,000 additional newborn lives through high coverage of skilled childbirth care, and save 310,000 lives through postnatal care [13]. Early initiation and completion of the continuum could greatly help in solving challenges and improve the health and survival of women, newborns, and children worldwide [1].

However, studies across Africa continue to reveal very low completion rates for the continuum of care [1, $14,15]$. These low rates in the trends have been associated with socio-economic factors like educational status, residence, distance from the health facility, woman's decision making power, early initiation of ANC, wealth index, media access, skills of health workers, health system supports, and presence of delivery fees $[5,13,16]$. These continue to affect the continuity of the continuum and pose setbacks to the fight against maternal and neonatal mortality especially in low- and middle-income countries. Analysis of the level of completion of the continuum has proven an effective approach in understanding $\mathrm{MCH}$ trends for any particular country. Therefore, this study is aimed at assessing the level and determinants of completion of the continuum of care for maternal and newborn health in Sierra Leone, using data from the most recent national demographic health survey of 2019. 


\section{Methods}

\section{Study design and sampling methods}

The Sierra Leone Demographic and Health Surveys (SLDHS) are cross-sectional surveys that are periodically conducted to obtain information on demographic, health and nutritional indicators of nonelderly adults and children. SLDHS was implemented by Statistics Sierra Leone (Stats SL) with technical assistance from ICF intern through the DHS Program and funded by the United States Agency for International Development (USAID). The latest survey was conducted over a four month period between 15th May 2019 and 31st August 2019 [10]. This national survey used stratified, two-stage cluster sampling design to obtain a representative sample of 13,872 households [10]. Weighted data was used to account for the unequal probability sampling in different strata. A detailed explanation of the sampling process is available elsewhere [10]. Women aged 15-49 years who were either permanent residents or visitors who had stayed in the selected households the night before the survey were eligible for interviews with a total of 15,574 women who were interviewed. Secondary analysis included women aged 15 to 49 years who had a live birth within five years preceding the survey (with the most recent birth being considered) and were either permanent residents or slept in the selected household the night preceding the survey. Out of the total weighted sample of 15,574 women in the data set, only 7,326 had given birth

within five years preceding the survey (as shown in Table 1). Of the 7,326 women, 112 (1.5\%) women had missing data on ANC initiation timing leading to a total of 7,214 women who were considered for logistic regression analysis (as shown in Table 3). The Demographic and Health Survey datasets are freely available to the public however researchers must first register with MEASURE DHS and then submit a request before accessing them. Written permission to access the whole ZDHS database was obtained through DHS program website [17].

\section{Variables}

\section{Outcome variables}

Complete continuum of maternal and newborn healthcare was the outcome variable and was constructed into a binary variable with complete coded as 1 and incomplete coded as 0 . Complete continuum of maternal and newborn healthcare was considered when a woman reported having had all the three conditions/states:

1. Had at least eight ANC contacts during the most recent childbirth.

2. Skilled birth attendance

3. Mother and baby had at least one postnatal check-up within six weeks after childbirth $[18,19]$

\section{Independent variables}


Eighteen independent variables were categorized into women and household characteristics, and were chosen basing on previous studies [20-22] and availability in the SLDHS database.

Household characteristics. Wealth index of household (categorized into quintiles: richest, richer, middle poorer and poorest), type of residence (urban and rural), and region that included the official five regions in the SLDHS (western, eastern, southern, northwestern and northern), household size (less than 7 and, 7 and above) and sex of household head (male and female) Wealth index is a measure of relative household economic status and was calculated by DHS from information on household asset ownership using principal component analysis [10, 23].

Women's characteristics. Age (15-24, 25-34, and 35-49 years), level of education (no education, primary, secondary, and tertiary), exposure to newspapers/magazines, internet, radio and TV (yes and no), parity (1, 2-4 and 5 and above), ANC initiation timing (first trimester and after first trimester), marital status (married and not married), working status (working and not working) and decision making for seeking healthcare (involved and not involved). Religion was categorized as Islam and Christianity and others while problems seeking permission and distance to health facility were categorized as big problem and no big problem. In the questionnaire, seeking permission to access healthcare and distance to health facility had three original responses: no problem, no big problem and big problem. However, none of the study participants reported no problem hence we only had two responses.

\section{Statistical analysis}

In order to account for the multi-stage cluster study design, complex sample package of SPSS (version 25.0) statistical software was used. Analysis was carried out based on the weighted count to account for the unequal probability sampling in different strata and to ensure representativeness of the survey results at the national and regional level.

Before multivariable logistic regression analysis, independent variables were assessed for their association with $\mathrm{CoC}$ utilisation using bivariable logistic regression analysis and we presented the crude odds ratio (COR), 95\% confidence interval $(\mathrm{Cl})$ and p-values. Independent variables associated with COC utilisation from literature and those with a $p$-value $\leq 0.25$ at the bivariable level, and not strongly collinear (considered variance inflation factor less than 3 ) with other independent variables were considered for multivariable logistic regression to assess the independent effect of each variable on the $\mathrm{CoC}$ utilisation. Adjusted odds ratios (aOR), 95\% confidence intervals (Cl) and p-values were calculated with statistical significance level set at $p$-value $<0.05$.

\section{Results}

A total of 7,326 women were included in the analysis (Table 1). Of these, 734 (10.0\%) (95\% Cl: 9.6-11.0) had complete continuum of care (further details in Table 2). Majority of the women resided in rural areas (61.9\%), had less than eight ANC contacts (78.0\%), had had skilled birth attendance (88.3\%), had a postnatal check after delivery $(90.7 \%)$, belonged to Islam $(78.7 \%)$, had no education $(52.7 \%)$, resided in 
male headed households (75.3\%), were married (81.9\%), working (77\%) and aged between 20 and 34 years $(65.9 \%)$. Most women had limited exposure to mass media with $57.7 \%$ of women not exposed to radio, $76.2 \%$ not exposed to TV, $89.9 \%$ not using internet and $94.5 \%$ not exposed to newspapers. The mean age and household size were $28.97 \pm 7.25$ years and $6.93 \pm 3.45$ members respectively. 
Table 1

Socio-demographic characteristics of women in Sierra Leone as per the 2019 SLDHS

\begin{tabular}{|c|c|c|}
\hline Characteristics & $\mathrm{N}=7,326$ & $\%$ \\
\hline \multicolumn{3}{|l|}{ Age } \\
\hline 15 to 19 & 598 & 8.2 \\
\hline 20 to 34 & 4830 & 65.9 \\
\hline 35 to 49 & 1898 & 25.9 \\
\hline \multicolumn{3}{|l|}{ Residence } \\
\hline Urban & 2795 & 38.1 \\
\hline Rural & 4531 & 61.9 \\
\hline \multicolumn{3}{|l|}{ Region } \\
\hline Western & 1479 & 20.2 \\
\hline Eastern & 1542 & 21.0 \\
\hline Northwestern & 1380 & 18.8 \\
\hline Northern & 1433 & 19.6 \\
\hline Southern & 1492 & 20.4 \\
\hline \multicolumn{3}{|l|}{ Religion } \\
\hline Islam & 5766 & 78.7 \\
\hline Christianity and others & 1560 & 21.3 \\
\hline \multicolumn{3}{|l|}{ Sex household head } \\
\hline Male & 5520 & 75.3 \\
\hline Female & 1806 & 24.7 \\
\hline \multicolumn{3}{|l|}{ Household Size } \\
\hline 7 and above & 3319 & 45.3 \\
\hline Less than 7 & 4007 & 54.7 \\
\hline \multicolumn{3}{|l|}{ Working status } \\
\hline Not working & 1683 & 23.0 \\
\hline Working & 5643 & 77.0 \\
\hline
\end{tabular}

$a_{=}$missing $112(1.5 \%)$ respondents 


\begin{tabular}{|lll|}
\hline Characteristics & $\mathrm{N}=7,326$ & $\%$ \\
\hline Marital status & & \\
\hline Not married & 1329 & 18.1 \\
\hline Married & 5997 & 81.9 \\
\hline Education Level & & \\
\hline No Education & 3857 & 52.7 \\
\hline Primary Education & 1033 & 14.1 \\
\hline Secondary Education & 2214 & 30.2 \\
\hline Tertiary & 221 & 3.0 \\
\hline Wealth Index & & \\
\hline Poorest & 1587 & 21.7 \\
\hline Poorer & 1551 & 21.1 \\
\hline Middle & 1487 & 20.3 \\
\hline Richer & 1441 & 19.7 \\
\hline Richest & 1259 & 17.2 \\
\hline ANC initiation timing & & \\
\hline First trimester & & 57.7 \\
\hline After first trimester & 4224 & 54.5 \\
\hline Parity & 3214 & 44.6 \\
\hline 1 & 4000 & 55.4 \\
\hline $2-4$ & & \\
\hline 5 and above & 1323 & 18.1 \\
\hline Exposure to newspapers & & \\
\hline No & & \\
\hline Yes & & 54.1 \\
\hline Exposure to Radio & & \\
\hline
\end{tabular}

${ }^{a}=$ missing $112(1.5 \%)$ respondents 


\begin{tabular}{|lll|}
\hline Characteristics & $\mathrm{N}=7,326$ & $\%$ \\
\hline Yes & 3102 & 42.3 \\
\hline Exposure to TV & & \\
\hline No & 5579 & 76.2 \\
\hline Yes & 1747 & 23.8 \\
\hline Internet use & & \\
\hline No & 6586 & 89.9 \\
\hline Yes & 740 & 10.1 \\
\hline Permission to access healthcare & & \\
\hline Big problem & 1827 & 24.9 \\
\hline Not big problem & 5499 & 75.1 \\
\hline Distance to health facility & & \\
\hline Big problem & 3454 & 47.1 \\
\hline Not big problem & 3872 & 52.9 \\
\hline a missing 112 (1.5\%) respondents & \\
\hline
\end{tabular}


Table 2

Utilisation of the different components of continuum of care

\begin{tabular}{|llll|}
\hline Service & $\begin{array}{l}\text { Frequency } \\
\text { N=7,326 }\end{array}$ & & $95 \%$ Cl \\
\hline 8 or more ANC contacts & 1610 & 22.0 & $21.3-23.1$ \\
\hline Skilled birth attendance & 6468 & 88.3 & $87.9-89.4$ \\
\hline Postnatal Care & & & \\
\hline Maternal PNC at health facility discharge ${ }^{\text {a }}$ & 5706 & 92.3 & $91.7-93.0$ \\
\hline Maternal PNC after discharge from health facility & 2715 & 37.1 & $35.9-38.1$ \\
\hline Maternal PNC (at least one of the above) & 6646 & 90.7 & $90.2-91.5$ \\
\hline Neonatal PNC at health facility discharge ${ }^{a}$ & 5735 & 92.8 & $92.1-93.4$ \\
\hline Neonatal PNC after discharge from health facility & 3329 & 45.5 & $44.6-46.8$ \\
\hline Neonatal PNC (at least one of the above) & 6625 & 90.4 & $89.9-91.2$ \\
\hline Both maternal and neonatal PNC & 6274 & 85.6 & $85.0-86.6$ \\
\hline Continuum of care & 1311 & 17.9 & $17.4-19.1$ \\
\hline a Misssing 1143 & & & \\
\hline
\end{tabular}

\section{Factors associated with CoC utilisation}

After adjusting for other variables, factors that were significantly associated with $\mathrm{COC}$ utilisation were; having started ANC within first trimester (aOR 1.71, 95\% Cl: 1.46-2.00), belonging to the Southern region (aOR 1.85, 95\% Cl: 1.23-2.80), belonging to richer wealth quintile (aOR 1.76, 95\% Cl: 1.27-2.44), using internet (aOR 1.49, 95\% Cl: 1.12-1.98) and having no big problems seeking permission to access healthcare (aOR 1.34, 95\% Cl: 1.06-1.69). 
Table 2

Factors associated with CoC utilisation in Sierra Leone as per the 2019 SLDHS

\begin{tabular}{|c|c|c|c|c|c|c|}
\hline \multirow[b]{2}{*}{ Characteristics } & \multicolumn{3}{|c|}{$\begin{array}{l}\text { Cross tabulation } \\
\mathrm{N}=7,326\end{array}$} & \multicolumn{3}{|c|}{$\begin{array}{l}\text { Logistic regression } \\
N=7,214\end{array}$} \\
\hline & $\begin{array}{l}\text { No COC } \\
n(\%)\end{array}$ & $\begin{array}{l}\text { Yes, CoC } \\
\text { n (\%) }\end{array}$ & $\begin{array}{l}\mathrm{P}- \\
\text { value }\end{array}$ & $\begin{array}{l}\text { Crude } \\
\text { model } \\
\mathrm{cOR}(95 \% \\
\mathrm{Cl})\end{array}$ & $\begin{array}{l}\text { P- } \\
\text { value }\end{array}$ & $\begin{array}{l}\begin{array}{l}\text { Adjusted } \\
\text { model }\end{array} \\
\text { aOR (95\% } \\
\mathrm{Cl})\end{array}$ \\
\hline Visited by fieldworker & & & 0.403 & & 0.403 & \\
\hline No & $\begin{array}{l}4173 \\
(69.4)\end{array}$ & $\begin{array}{l}886 \\
(67.6)\end{array}$ & & 1 & & - \\
\hline Yes & $\begin{array}{l}1842 \\
(30.6)\end{array}$ & $\begin{array}{l}425 \\
(32.4)\end{array}$ & & $\begin{array}{l}1.09 \\
1.32)\end{array}$ & & \\
\hline ANC timing ${ }^{a}$ & & & $<0.001$ & & $<0.001$ & \\
\hline Above first trimester & $\begin{array}{l}2480 \\
(42.0)\end{array}$ & $\begin{array}{l}733 \\
(55.9)\end{array}$ & & 1 & & 1 \\
\hline Within first trimester & $\begin{array}{l}3422 \\
(58.0)\end{array}$ & $\begin{array}{l}578 \\
(44.1)\end{array}$ & & $\begin{array}{l}1.75 \\
2.04)\end{array}$ & & $\begin{array}{l}1.71(1.46- \\
2.00)\end{array}$ \\
\hline Age & & & 0.735 & & 0.771 & \\
\hline 35 to 49 & $\begin{array}{l}1548 \\
(25.7)\end{array}$ & $\begin{array}{l}350 \\
(26.7)\end{array}$ & & 1 & & - \\
\hline 20 to 34 & $\begin{array}{l}3971 \\
(66.0)\end{array}$ & $\begin{array}{l}860 \\
(65.6)\end{array}$ & & $\begin{array}{l}0.96(0.83- \\
1.11)\end{array}$ & 0.572 & \\
\hline 15 to 19 & $496(8.3)$ & $101(7.7)$ & & $\begin{array}{l}0.90(0.67- \\
1.22)\end{array}$ & 0.504 & \\
\hline Residence & & & 0.150 & & 0.151 & \\
\hline Rural & $\begin{array}{l}3762 \\
(62.5)\end{array}$ & $\begin{array}{l}769 \\
(58.7)\end{array}$ & & 1 & & 1 \\
\hline Urban & $\begin{array}{l}2253 \\
(37.5)\end{array}$ & $\begin{array}{l}542 \\
(41.3)\end{array}$ & & $\begin{array}{l}1.17(0.94- \\
1.47)\end{array}$ & & $\begin{array}{l}0.99(0.70- \\
1.41)\end{array}$ \\
\hline Region & & & 0.003 & & 0.001 & \\
\hline Western & $\begin{array}{l}1227 \\
(20.4)\end{array}$ & $\begin{array}{l}252 \\
(19.2)\end{array}$ & & 1 & & 1 \\
\hline Southern & $\begin{array}{l}1159 \\
(19.3)\end{array}$ & $\begin{array}{l}333 \\
(25.4)\end{array}$ & & $\begin{array}{l}1.40(1.01- \\
1.96)\end{array}$ & 0.048 & $\begin{array}{l}1.85(1.23- \\
2.80)\end{array}$ \\
\hline
\end{tabular}

$a_{=}$missing $112(1.5 \%)$ respondents, Bold: significant at $<0.05$ 


\begin{tabular}{|c|c|c|c|c|c|c|}
\hline \multirow{2}{*}{ Northwestern } & \multicolumn{2}{|c|}{$\begin{array}{l}\text { Cross tabulation } \\
\mathrm{N}=7,326\end{array}$} & & \multicolumn{3}{|c|}{$\begin{array}{l}\text { Logistic regression } \\
\mathrm{N}=7,214\end{array}$} \\
\hline & $\begin{array}{l}1167 \\
(19.4)\end{array}$ & $\begin{array}{l}213 \\
(16.2)\end{array}$ & & $\begin{array}{l}0.89(0.63- \\
1.27)\end{array}$ & 0.520 & $\begin{array}{l}1.16(0.74- \\
1.82)\end{array}$ \\
\hline Northern & $\begin{array}{l}1237 \\
(20.6)\end{array}$ & $\begin{array}{l}197 \\
(15.0)\end{array}$ & & $\begin{array}{l}0.78(0.53- \\
1.13)\end{array}$ & 0.185 & $\begin{array}{l}0.93(0.61- \\
1.44)\end{array}$ \\
\hline Eastern & $\begin{array}{l}1225 \\
(20.3)\end{array}$ & $\begin{array}{l}316 \\
(24.1)\end{array}$ & & $\begin{array}{l}1.26(0.87- \\
1.83)\end{array}$ & 0.224 & $\begin{array}{l}1.52(0.99- \\
2.33)\end{array}$ \\
\hline Religion & & & 0.038 & & 0.038 & \\
\hline Islam & $\begin{array}{l}4773 \\
(79.3)\end{array}$ & $\begin{array}{l}994 \\
(75.8)\end{array}$ & & 1 & & 1 \\
\hline Christianity and others & $\begin{array}{l}1242 \\
(20.7)\end{array}$ & $\begin{array}{l}317 \\
(24.2)\end{array}$ & & $\begin{array}{l}1.23(1.01- \\
1.49)\end{array}$ & & $\begin{array}{l}1.07(0.87- \\
1.32)\end{array}$ \\
\hline Sex household head & & & 0.180 & & 0.180 & \\
\hline Male & $\begin{array}{l}4557 \\
(75.8)\end{array}$ & $\begin{array}{l}963 \\
(73.5)\end{array}$ & & 1 & & 1 \\
\hline Female & $\begin{array}{l}1458 \\
(24.2)\end{array}$ & $\begin{array}{l}348 \\
(26.5)\end{array}$ & & $\begin{array}{l}1.13(0.95- \\
1.35)\end{array}$ & & $\begin{array}{l}1.06(0.87- \\
1.28)\end{array}$ \\
\hline Household Size & & & 0.125 & & 0.125 & \\
\hline 7 and above & $\begin{array}{l}2755 \\
(45.8)\end{array}$ & $\begin{array}{l}564 \\
(43.0)\end{array}$ & & 1 & & 1 \\
\hline Less than 7 & $\begin{array}{l}3260 \\
(54.2)\end{array}$ & $\begin{array}{l}747 \\
(57.0)\end{array}$ & & $\begin{array}{l}1.12(0.97- \\
1.30)\end{array}$ & & $\begin{array}{l}1.11(0.95- \\
1.29)\end{array}$ \\
\hline Working status & & & 0.372 & & 0.373 & \\
\hline Not working & $\begin{array}{l}1396 \\
(23.2)\end{array}$ & $\begin{array}{l}287 \\
(21.9)\end{array}$ & & 1 & & - \\
\hline Working & $\begin{array}{l}4619 \\
(76.8)\end{array}$ & $\begin{array}{l}1024 \\
(78.1)\end{array}$ & & $\begin{array}{l}1.08(0.91- \\
1.28)\end{array}$ & & \\
\hline Marital status & & & 0.214 & & 0.215 & \\
\hline Not married & $\begin{array}{l}1073 \\
(17.8)\end{array}$ & $\begin{array}{l}256 \\
(19.5)\end{array}$ & & 1 & & 1 \\
\hline Married & $\begin{array}{l}4942 \\
(82.2)\end{array}$ & $\begin{array}{l}1055 \\
(80.5)\end{array}$ & & $\begin{array}{l}0.90(0.76- \\
1.07)\end{array}$ & & $\begin{array}{l}1.05(0.87- \\
1.26)\end{array}$ \\
\hline Education Level & & & $<0.001$ & & $<0.001$ & \\
\hline
\end{tabular}

$a_{=}$missing $112(1.5 \%)$ respondents, Bold: significant at $<0.05$ 


\begin{tabular}{|c|c|c|c|c|c|c|}
\hline \multirow{2}{*}{ No Education } & \multicolumn{3}{|c|}{$\begin{array}{l}\text { Cross tabulation } \\
\mathrm{N}=7,326\end{array}$} & \multicolumn{3}{|c|}{$\begin{array}{l}\text { Logistic regression } \\
\mathrm{N}=7,214\end{array}$} \\
\hline & $\begin{array}{l}3226 \\
(53.6)\end{array}$ & $\begin{array}{l}632 \\
(48.2)\end{array}$ & & 1 & & 1 \\
\hline Primary Education & $\begin{array}{l}829 \\
(13.8)\end{array}$ & $\begin{array}{l}204 \\
(15.6)\end{array}$ & & $\begin{array}{l}1.26(1.03- \\
1.54)\end{array}$ & 0.027 & $\begin{array}{l}1.13(0.91- \\
1.39)\end{array}$ \\
\hline Secondary Education & $\begin{array}{l}1801 \\
(29.9)\end{array}$ & $\begin{array}{l}413 \\
(31.5)\end{array}$ & & $\begin{array}{l}1.17(0.99- \\
1.39)\end{array}$ & 0.066 & $\begin{array}{l}0.93(0.77- \\
1.13)\end{array}$ \\
\hline Tertiary & $159(2.6)$ & $62(4.7)$ & & $\begin{array}{l}1.98(1.43- \\
2.75)\end{array}$ & $<0.001$ & $\begin{array}{l}1.26(0.84- \\
1.90)\end{array}$ \\
\hline Wealth Index & & & 0.004 & & 0.003 & \\
\hline Poorest & $\begin{array}{l}1358 \\
(22.6)\end{array}$ & $\begin{array}{l}229 \\
(17.5)\end{array}$ & & 1 & & 1 \\
\hline Poorer & $\begin{array}{l}1288 \\
(21.4)\end{array}$ & $\begin{array}{l}263 \\
(20.1)\end{array}$ & & $\begin{array}{l}1.21(0.98- \\
1.50)\end{array}$ & 0.082 & $\begin{array}{l}1.28(1.02- \\
1.60)\end{array}$ \\
\hline Middle & $\begin{array}{l}1206 \\
(20.0)\end{array}$ & $\begin{array}{l}281 \\
(21.4)\end{array}$ & & $\begin{array}{l}1.38(1.12- \\
1.71)\end{array}$ & 0.003 & $\begin{array}{l}1.45(1.17- \\
1.79)\end{array}$ \\
\hline Richer & $\begin{array}{l}1127 \\
(18.7)\end{array}$ & $\begin{array}{l}315 \\
(24.0)\end{array}$ & & $\begin{array}{l}1.66(1.27- \\
2.16)\end{array}$ & $<0.001$ & $\begin{array}{l}1.76(1.27- \\
2.44)\end{array}$ \\
\hline Richest & $\begin{array}{l}1036 \\
(17.3)\end{array}$ & $\begin{array}{l}223 \\
(17.0)\end{array}$ & & $\begin{array}{l}1.27(0.93- \\
1.74)\end{array}$ & 0.134 & $\begin{array}{l}1.35(0.86- \\
2.10)\end{array}$ \\
\hline Parity & & & 0.073 & & 0.048 & \\
\hline 5 and above & $\begin{array}{l}1118 \\
(18.6)\end{array}$ & $\begin{array}{l}204 \\
(15.6)\end{array}$ & & 1 & & 1 \\
\hline $2-4$ & $\begin{array}{l}3282 \\
(54.6)\end{array}$ & $\begin{array}{l}733 \\
(55.9)\end{array}$ & & $\begin{array}{l}1.22(1.02- \\
1.46)\end{array}$ & 0.028 & $\begin{array}{l}1.16(0.95- \\
1.41)\end{array}$ \\
\hline 1 & $\begin{array}{l}1615 \\
(27.2)\end{array}$ & $\begin{array}{l}374 \\
(28.5)\end{array}$ & & $\begin{array}{l}1.27(1.03- \\
1.56)\end{array}$ & 0.025 & $\begin{array}{l}1.17(0.92- \\
1.49)\end{array}$ \\
\hline Newspapers exposure & & & 0.143 & & 0.143 & \\
\hline No & $\begin{array}{l}5696 \\
(94.7)\end{array}$ & $\begin{array}{l}1225 \\
(93.4)\end{array}$ & & 1 & & 1 \\
\hline Yes & $319(5.3)$ & $86(6.6)$ & & $\begin{array}{l}1.25(0.93- \\
1.68)\end{array}$ & & $\begin{array}{l}0.98(0.72- \\
1.34)\end{array}$ \\
\hline Exposure to Radio & & & 0.091 & & 0.091 & \\
\hline
\end{tabular}

a = missing $112(1.5 \%)$ respondents, Bold: significant at $<0.05$ 


\begin{tabular}{|c|c|c|c|c|c|c|}
\hline & $\begin{array}{l}\text { Cross tab } \\
\mathrm{N}=7,326\end{array}$ & ation & & $\begin{array}{l}\text { Logistic reg } \\
N=7,214\end{array}$ & sion & \\
\hline No & $\begin{array}{l}3506 \\
(58.3)\end{array}$ & $\begin{array}{l}718 \\
(54.7)\end{array}$ & & 1 & & 1 \\
\hline Yes & $\begin{array}{l}2509 \\
(41.7)\end{array}$ & $\begin{array}{l}593 \\
(45.3)\end{array}$ & & $\begin{array}{l}1.16(0.98- \\
1.37)\end{array}$ & & $\begin{array}{l}1.05(0.88- \\
1.25)\end{array}$ \\
\hline Exposure to TV & & & 0.391 & & 0.391 & \\
\hline No & $\begin{array}{l}4562 \\
(75.8)\end{array}$ & $\begin{array}{l}1017 \\
(77.6)\end{array}$ & & 1 & & - \\
\hline Yes & $\begin{array}{l}1453 \\
(24.2)\end{array}$ & $\begin{array}{l}294 \\
(22.4)\end{array}$ & & $\begin{array}{l}0.90(0.73- \\
1.13)\end{array}$ & & \\
\hline Internet use & & & $<0.001$ & & $<0.001$ & \\
\hline No & $\begin{array}{l}5461 \\
(90.8)\end{array}$ & $\begin{array}{l}1125 \\
(85.8)\end{array}$ & & 1 & & 1 \\
\hline Yes & $554(9.2)$ & $\begin{array}{l}186 \\
(14.2)\end{array}$ & & $\begin{array}{l}1.63(1.28- \\
2.08)\end{array}$ & & $\begin{array}{l}1.49(1.12- \\
1.98)\end{array}$ \\
\hline $\begin{array}{l}\text { Permission to access } \\
\text { healthcare }\end{array}$ & & & 0.020 & & 0.020 & \\
\hline Big problem & $\begin{array}{l}1549 \\
(25.8)\end{array}$ & $\begin{array}{l}277 \\
(21.1)\end{array}$ & & 1 & & 1 \\
\hline Not big problem & $\begin{array}{l}4466 \\
(74.2)\end{array}$ & $\begin{array}{l}1034 \\
(78.9)\end{array}$ & & $\begin{array}{l}1.29(1.04- \\
1.61)^{(1.04}\end{array}$ & & $\begin{array}{l}1.34(1.06- \\
1.69)\end{array}$ \\
\hline Distance to health facility & & & 0.802 & & 0.802 & \\
\hline Big problem & $\begin{array}{l}2829 \\
(47.0)\end{array}$ & $\begin{array}{l}625 \\
(47.7)\end{array}$ & & 1 & & - \\
\hline Not big problem & $\begin{array}{l}3186 \\
(53.0)\end{array}$ & $\begin{array}{l}686 \\
(52.3)\end{array}$ & & $\begin{array}{l}0.98(0.80- \\
1.19)\end{array}$ & & \\
\hline
\end{tabular}

\section{Discussion}

This study focused on the level of $\mathrm{CoC}$ in Sierra Leone considering the utilization of three major aspects of maternal and newborn health such as eight ANC contacts, skilled birth attendance, maternal and neonatal postnatal care as well as their association with various socio-demographic and household related factors. 
This study revealed that most women and their newborns did not receive maternal and newborn health services continuously. As per our results only $22 \%$ utilized 8 or more ANC contacts, $88 \%$ had skilled birth attendance while $90.7 \%$ and $90.4 \%$ of mothers and neonates utilized PNC respectively. However, only $17.9 \%$ of women in Sierra Leone utilized complete $\mathrm{CoC}$ for maternal and newborn at all three levels. This low completion rate of $\mathrm{CoC}$ in the country could imply an increased likelihood of maternal complications and neonatal mortality, as many women and their children miss proven interventions at various contact points of the continuum. The results clearly showed that the new WHO eight ANC contacts model is yet to be institutionalized among women of childbearing age in Sierra Leone. Women attended ANC after first trimester, from southern region, richer, used internet and had no big problem seeking permission were significantly associated with utilization of complete $\mathrm{CoC}$ for maternal and newborn health services at the three levels.

The magnitude of $\mathrm{CoC}$ is higher than those of studies conducted in a rural district of Lao People's Democratic Republic [24] Ghana [14] and Cambodia [25], as only $6.8 \%, 7.9 \%$ and $5.0 \%$ continued to receive $\mathrm{CoC}$ for maternal and newborn services at the three levels respectively. It is lower than those of studies conducted in Zambia [1], Egypt (50\%) [19] and Pakistan (27\%) [12]. These countries show that the disparities in utilization of complete continuum of care is because of shortages in human, financial and inadequate health system infrastructure, even though different ways of assessing continuum of care ware used. The low level in Sierra Leone could be explained by the impact of Ebola outbreak on reproductive health services among other socio-demographic factors [26].

Timing of ANC initiation was associated with utilization of complete CoC for maternal and newborn health services. Women who had their first ANC visit after the first trimester had less odds of utilizing complete continuum of care compared to those who initiated ANC in their first trimester which similar finding was shown in Zambia [1]. A plausible explanation is that, the time at which first ANC visit is done has the utmost importance to ensure optimal health effects for both women and children [1]. This enables women to have more ANC contacts which offer an opportunity to establish baseline information on her general wellbeing, maternal and child counselling and education and creates good rapport with her health care provider hence increasing the chances of linking to the other components of continuum of care and promoting completion [27, 28]. Kinney et al. while analyzing data from sub-Saharan Africa showed that women who had initiated ANC from the first trimester had lower odds of maternal complications and mortality [29]. Therefore, there is need for stakeholders to promote timely ANC initiation as a way of ensuring increased $\mathrm{CoC}$ utilization.

Women from the Southern region demonstrated a more likelihood of utilizing the complete continuum of care package compared to those from the Western region. Similarly, studies conducted in Ghana, Pakistan and Zambia documented the role of regional disparities in explaining the utilization of complete $\operatorname{CoC}[1,12,15]$. Post-conflict in Sierra Leone, the health system has been described as fragile [26]. The Western region having a high concentration of skilled birth attendants with higher social amenities due to the high economic development compared to other regions [30, 31], one would expect higher CoC utilization. However, the Western region has increasingly registered a high number of urban poor and high 
standards of living which has led to inequitable access and affordability of social amenities and services including public and private health facilities, which negatively affects access to healthcare [32, 33]. In addition, the Western region being highly developed has led to health non-governmental organizations implementing maternal and child health programs to concentrate on other regions such as the Southern region could also partly explain this finding.

Women belonging to richer households were positively associated with complete continuum of maternal and newborn care, a finding consistent with several other studies including one from Cambodia [34] and another from [35]. The direct and indirect costs incurred when accessing care limit the ability of women from poor households to access maternal health services [3].This implies that lower levels of wealth can also be a barrier to accessing maternal health services hence multi-sectoral actors and different attentions are needed to eliminate financial barriers to improve the continuity of maternity care in Sierra Leone.

Women who used internet were associated with higher odds of utilizing complete continuum of care compared to those who did not use. Similarly, a study conducted in an urban city in Nigeria suggests that internet is more popular compared to television and radio due to its high degree of interactivity, confidential and easily accessible especially on sensitive topics like reproductive health [36]. A review article revealed the important role the internet plays for women during pregnancy as it helps them to seek information in their early days of pregnancy and to make informed decisions [37]. The reasons behind the popularity of internet use in Sierra Leone could be that the internet provides various platforms to search information on maternal health as needed and equips the women with maternal health information on the importance of utilizing the continuum of care package [38].

Women who did not have a big problem in seeking permission to access a health facility were more likely to complete the three levels of continuum of care compared to those who had a big problem. High decision-making power has been shown to increase ANC contacts in Benin [39]. Increased decisionmaking power results from increased autonomy and empowerment among women, which depicts more access to health information and financial independence such that decisive decisions about her health are solely made by her [40].

\section{Strengths and Limitations.}

The novelty of this study stems from the fact that, it is the first of its kind to have investigated continuum of care considering the current WHO guidelines in Sierra Leone. The study made use of cross-sectional survey data, which reflect the views of women aged 15-49 across the entire country and as such, the results and conclusions are based on a nationally representative survey. However, the study had some limitations. First, the data were collected based on women's self-reports. This may lead to recall bias, information bias or social desirability bias. Second, the cross-sectional nature of the study does not permit causal inferences to be made. Lastly, the study included women who gave birth within five years preceding the survey (2015-2019) yet the WHO ANC guidelines were introduced in 2016 so considering the 8 ANC recommended contacts risks a lower prevalence of utilization of these ANC contacts. 


\section{Conclusion}

The low completion rate of the entire continuum of maternal care shown in this study indicates that women in Sierra Leone are not receiving the maximum possible health benefits from the existing health services. The predictors of completion of $\mathrm{CoC}$ have been demonstrated to operate at various levels individual, household and community hence highlighting a need to contextualize efforts. Efforts to promote the use of maternal health services need to focus on regional differences in utilizing CoC, women with no access to internet, those with low autonomy, and those with low socio-economic status. Further research is needed in investigating and understanding why women do not complete the continuum of maternity care. Besides, programs promoting maternal health utilization should emphasize accessing the full package rather than only specific components.

\section{Abbreviations}

EA Enumeration area

AOR Adjusted Odds Ratio

$\mathrm{Cl}$ Confidence Interval

COR Crude Odds Ratio

DHS Demographic Health Survey

SLDHS Sierra Leone Demographic Health Survey

OR Odds Ratio

SD Standard Deviation

WHO World Health Organization

ANC Antenatal care

PNC Postnatal care

SBA Skilled Birth Attendance

CoC Continuum of Care

SPSS Statistical Package for Social Science

\section{Declarations}

Acknowledgements 
We thank the DHS program for making the data available for this study.

\section{Funding information}

No funding was obtained for this study.

\section{Availability of data and materials}

The data set used is openly available upon permission from MEASURE DHS website (URL: https://www.dhsprogram.com/data/available-datasets.cfm). However, authors are not authorized to share this data set to the public but anyone interested in the data set can seek it with written permission from MEASURE DHS website (URL: https://www.dhsprogram.com/data/available-datasets.cfm).

\section{Author contributions}

QS Conceived the idea, drafted the manuscript, performed analysis, interpreted the results and drafted the subsequent versions of the manuscript. KK, MWM, LMM, LN and RKM reviewed the first draft and drafted the subsequent versions of the manuscript. All authors read and approved the final manuscript.

\section{Ethics approval and consent to participate}

High international ethical standards are ensured during MEASURE DHS surveys and the study protocol is performed in accordance with the relevant guidelines. The SLDHS 2019 survey protocol was reviewed and approved by the Sierra Leone Ethics and Scientific Review Committee and the ICF Institutional Review Board. Written informed consent was obtained from human participants and written informed consent was also obtained from legally authorized representatives of minor participants.

\section{Consent for publication}

Not applicable.

\section{Competing interests}

All authors declare that they have no competing interests.

\section{References}

1. Sserwanja Q, Musaba MW, Mutisya LM, Olal E, Mukunya D: Continuum of maternity care in Zambia: a national representative survey. BMC pregnancy and childbirth 2021, 21(1):604.

2. World Health Organization. Health in 2015: From MDGs to SDGs, 69-98. https://www.who.int/gho/publications/mdgs-sdgs/MDGs-SDGs2015_chapter4.pdf?ua=1.

3. Sserwanja Q, Mukunya D, Musaba MW, Kawuki J, Kitutu FE: Factors associated with health facility utilization during childbirth among 15 to 49-year-old women in Uganda: evidence from the Uganda demographic health survey 2016. BMC Health Services Research 2021, 21(1):1160. 
4. Alkema L, Zhang S, Chou D, Gemmill A, Moller A-B, Fat DM, Say L, Mathers C, Hogan D: A Bayesian approach to the global estimation of maternal mortality. The Annals of Applied Statistics 2017, 11(3):1245-1274, 1230.

5. Atnafu A, Kebede A, Misganaw B, Teshome DF, Biks GA, Demissie GD, Wolde HF, Gelaye KA, Yitayal $M$, Ayele TA et al: Determinants of the Continuum of Maternal Healthcare Services in Northwest Ethiopia: Findings from the Primary Health Care Project. Journal of Pregnancy 2020, 2020:4318197.

6. Hug L, Alexander M, You D, Alkema L, Estimation UNI-aGfCM: National, regional, and global levels and trends in neonatal mortality between 1990 and 2017, with scenario-based projections to 2030: a systematic analysis. Lancet Glob Health 2019, 7(6):e710-e720.

7. Assaf S, Winter R: Trends in maternal health indicators in Sierra Leone, 2008-2013. In: DHS Further Analysis Reports No 97. Rockville, Maryland, USA: ICF International; 2015.

8. Kanu JS, Tang Y, Liu Y: Assessment on the knowledge and reported practices of women on maternal and child health in rural Sierra Leone: a cross-sectional survey. PLoS One 2014, 9(8):e105936e105936.

9. Sharkey A, Yansaneh A, Bangura PS, Kabano A, Brady E, Yumkella F, Diaz T: Maternal and newborn care practices in Sierra Leone: a mixed methods study of four underserved districts. Health Policy and Planning 2016, 32(2):151-162.

10. Statistics Sierra Leone - StatsSL, ICF: Sierra Leone Demographic and Health Survey 2019. In. Freetown/Sierra Leone: StatsSL/ICF; 2020.

11. Jalloh MB, Bah AJ, James PB, Sevalie S, Hann K, Shmueli A: Impact of the free healthcare initiative on wealth-related inequity in the utilization of maternal \& child health services in Sierra Leone. $B M C$ health services research 2019, 19(1):352-352.

12. Iqbal S, Maqsood S, Zakar R, Zakar MZ, Fischer F: Continuum of care in maternal, newborn and child health in Pakistan: analysis of trends and determinants from 2006 to 2012. 2017, 17(1):189.

13. Tizazu MA, Sharew NT: Completing the Continuum of Maternity Care and Associated Factors in Debre Berhan Town, Amhara, Ethiopia, 2020. 2021, 14:21-32.

14. Shibanuma A, Yeji F, Okawa S, Mahama E, Kikuchi K: The coverage of continuum of care in maternal, newborn and child health: a cross-sectional study of woman-child pairs in Ghana. 2018, 3(4):e000786.

15. Yeji F, Shibanuma A, Oduro A, Debpuur C, Kikuchi K, Owusu-Agei S, Gyapong M, Okawa S, Ansah E, Asare GQ et al: Continuum of Care in a Maternal, Newborn and Child Health Program in Ghana: Low Completion Rate and Multiple Obstacle Factors. PLoS One 2015, 10(12):e0142849.

16. Sahoo KC, Negi S, Patel K, Mishra BK, Palo SK, Pati S: Challenges in Maternal and Child Health Services Delivery and Access during Pandemics or Public Health Disasters in Low-and MiddleIncome Countries: A Systematic Review. Healthcare 2021, 9(7):828.

17. DHS. The DHS program. https://www.dhsprogram.com/.

18. Iqbal S, Maqsood S, Zakar R, Zakar MZ, Fischer F. Continuum of care in maternal, newborn and child health in Pakistan: analysis of trends and determinants from 2006 to 2012. BMC Health Serv Res. 
2017;17(1):189. Published 2017 Mar 9. doi:10.1186/s12913-017-2111-9.

19. Hamed A F, Roshdy E, Sabry M. Egyptian status of continuum of care for maternal, newborn, and childhealth: Sohag Governorate as an example. Int. J. Med. Med. Sci. 2018; 7:6.

20. Muthuri SK, Francis CE, Wachira LJ, Leblanc AG, Sampson M, Onywera VO, Tremblay MS: Evidence of an overweight/obesity transition among school-aged children and youth in Sub-Saharan Africa: a systematic review. PLoS One 2014, 9(3):e92846.

21. Tchoubi S, Sobngwi-Tambekou J, Noubiap JJ, Asangbeh SL, Nkoum BA, Sobngwi E: Prevalence and Risk Factors of Overweight and Obesity among Children Aged 6-59 Months in Cameroon: A Multistage, Stratified Cluster Sampling Nationwide Survey. PLoS One 2015, 10(12):e0143215.

22. Danquah Fl, Ansu-Mensah M, Bawontuo V, Yeboah M, Udoh RH, Tahiru M, Kuupiel D: Risk factors and morbidities associated with childhood obesity in sub-Saharan Africa: a systematic scoping review. BMC Nutrition 2020, 6(1):37.

23. Rutstein SO, Staveteig S: Making the Demographic and Health Surveys Wealth Index comparable. In: DHS Methodological Reports No 9. Rockville, Maryland, USA: ICF International; 2014.

24. Sakuma S, Yasuoka J, Phongluxa K, Jimba M: Determinants of continuum of care for maternal, newborn, and child health services in rural Khammouane, Lao PDR. 2019, 14(4):e0215635.

25. Kikuchi K, Yasuoka J, Nanishi K, Ahmed A, Nohara Y, Nishikitani M, Yokota F, Mizutani T, Nakashima $\mathrm{N}$ : Postnatal care could be the key to improving the continuum of care in maternal and child health in Ratanakiri, Cambodia. PLoS One 2018, 13(6):e0198829.

26. Quaglio G, Tognon F, Finos L, Bome D, Sesay S, Kebbie A, Di Gennaro F, Camara BS, Marotta C, Pisani $\checkmark$ et al: Impact of Ebola outbreak on reproductive health services in a rural district of Sierra Leone: a prospective observational study. BMJ open 2019, 9(9):e029093-e029093.

27. Wang W, Hong R: Levels and determinants of continuum of care for maternal and newborn health in Cambodia-evidence from a population-based survey. BMC pregnancy and childbirth 2015, 15:62.

28. Moller AB, Newby H, Hanson C, Morgan A: Measures matter: A scoping review of maternal and newborn indicators. 2018, 13(10):e0204763.

29. Kinney MV, Kerber KJ, Black RE, Cohen B, Nkrumah F, Coovadia H, Nampala PM, Lawn JE, Axelson H, Bergh AM et al: Sub-Saharan Africa's mothers, newborns, and children: where and why do they die? PLoS medicine 2010, 7(6):e1000294.

30. Wurie HR, Samai M, Witter S: Retention of health workers in rural Sierra Leone: findings from life histories. Human resources for health 2016, 14:3-3.

31. Witter $S$, Wurie $H$, Bertone MP: The free health care initiative: how has it affected health workers in Sierra Leone? Health policy and planning 2016, 31(1):1-9.

32. Austin V, Holloway C, Ossul Vermehren I, Dumbuya A, Barbareschi G, Walker J: "Give Us the Chance to Be Part of You, We Want Our Voices to Be Heard": Assistive Technology as a Mediator of Participation in (Formal and Informal) Citizenship Activities for Persons with Disabilities Who Are Slum Dwellers in Freetown, Sierra Leone. International journal of environmental research and public health 2021, 18(11):5547. 
33. Osuteye E, Koroma B, Macarthy JM, Kamara SF, Conteh A: Fighting COVID-19 in Freetown, Sierra Leone: the critical role of community organisations in a growing pandemic. Open Health 2020, 1(1):51-63.

34. Chham S, Radovich E, Buffel V, Ir P, Wouters E: Determinants of the continuum of maternal health care in Cambodia: an analysis of the Cambodia demographic health survey 2014. BMC pregnancy and childbirth 2021, 21(1):410.

35. Sanogo NdA, Yaya S: Wealth Status, Health Insurance, and Maternal Health Care Utilization in Africa: Evidence from Gabon. BioMed Research International 2020, 2020:4036830.

36. Nwagwu WE: The Internet as a source of reproductive health information among adolescent girls in an urban city in Nigeria. BMC Public Health 2007, 7(1):354.

37. Javanmardi M, Noroozi M, Mostafavi F, Ashrafi-Rizi H: Internet Usage among Pregnant Women for Seeking Health Information: A Review Article. Iranian journal of nursing and midwifery research 2018, 23(2):79-86.

38. Khatiwada J, Muzembo BA, Wada K, Ikeda S: Dimensions of women's empowerment on access to skilled delivery services in Nepal. BMC pregnancy and childbirth 2020, 20(1):622.

39. Ekholuenetale $\mathrm{M}$, Nzoputam $\mathrm{Cl}$, Barrow A, Onikan A: Women's enlightenment and early antenatal care initiation are determining factors for the use of eight or more antenatal visits in Benin: further analysis of the Demographic and Health Survey. Journal of the Egyptian Public Health Association 2020, 95(1):13.

40. Yaya S, Bishwajit G, Ekholuenetale M, Shah V, Kadio B, Udenigwe O: Timing and adequate attendance of antenatal care visits among women in Ethiopia. PLoS One 2017, 12(9):e0184934e0184934. 